\title{
Los entrenadores como promotores de la cohesión del equipo
}

\author{
Coaches as promoters of team cohesion
}

\section{Os treinadores como promotores da coesáo de equipa}

\author{
Isabel Balaguer ${ }^{1 *}$, Isabel Castillo', Luis Ródenas ${ }^{2}$, Priscila Fabra ${ }^{2}$ y Joan L. Duda ${ }^{2}$ \\ ${ }^{1}$ Universitat de València, ${ }^{2}$ School of Sport, Exercise and Rehabilitation Sciences, Birmingham, UK
}

Resumen: El objetivo del presente trabajo fue triple: 1) Estudiar las relaciones entre el clima motivacional percibido creado por el entrenador y la cohesión, 2) Poner a prueba un modelo con la siguiente secuencia: dimensiones del clima motivacional percibido $\rightarrow$ motivación autodeterminada $\rightarrow$ dimensiones de la cohesión, y 3) Estudiar el papel mediador de la motivación autodeterminada entre el clima motivacional percibido y la cohesión. Ochocientos nueve jóvenes jugadores de fútbol (798 chicos y 11 chicas; $M$ $=11.49, D T=1.16)$ completaron un paquete de cuestionarios en los que se evaluaban las variables de interés al principio de la temporada. Las correlaciones bivariadas informaron de relaciones positivas entre la percepción del clima de implicación en la tarea y ambas dimensiones de cohesión (tarea y social), mientras que el clima de implicación en el ego presentó correlaciones negativas con la cohesión de tarea. Los resultados del modelo de ecuaciones estructurales informaron que el clima-tarea se asoció positivamente con la motivación autodeterminada, mientras que el clima-ego lo hizo en sentido negativo. La motivación autodeterminada se relacionó de forma positiva tanto con la cohesión de tarea como con la cohesión social. Finalmente, la motivación autodeterminada actuó como mediador parcial entre el clima de implicación en la tarea y ambas dimensiones de la cohesión, y de mediador total entre el clima de implicación en el ego y la cohesión (tarea y social). Se enfatizan las implicaciones de las conductas de los entrenadores para la construcción de la cohesión en los equipos y para el desarrollo de la calidad de la motivación.

Palabras clave: Adolescentes, fútbol, clima motivacional, calidad de la motivación.

Abstract: The objective of this study was threefold: 1) To determine the links between the perceived coach-created motivational climate and cohesion, 2) To test a model with the following sequence: Dimensions of the perceived coach-created motivational climate $\rightarrow$ self-determined motivation $\rightarrow$ Dimensions of cohesion, and 3) To examine the meditational role of self-determined motivation in the relationship between perceived coach-created motivational climate and cohesion. Eight hundred and night young footballers (789 boys and 11 girls; $M$ age $=11.49 \pm 1.16)$ completed a package of questionnaires assessing the variables of interest at the beginning of the season. Bivariate correlations revealed positive relations between perceptions of a task-involving climate and both task and social cohesion, while perceived ego-involving climate was negatively related to task cohesion. Structural equation modeling revealed perceptions of a task involving climate and ego involving climate were positively and negatively, respectively, related to self-determined motivation. Self-determined motivation was positively related with both task cohesion and social cohesion. Finally, self-determined motivation partially mediated the relationships between perceptions of a task involving climate and both dimensions of cohesion (task and social). Total mediation, however, was supported in the case of perceived ego involving climate and task and social cohesion. The implications of coach behaviours for team cohesion building and players quality of motivation are emphasized.

Key words: Adolescents, soccer, motivational climate, quality of motivation. Resumo: O objectivo do presente trabalho foi triplo: 1) Estudar as relaçóes entre o clima motivacional percebido criado pelo treinador e a coesão, 2) Testar um modelo com a seguinte sequência: dimensóes do clima motivacional percebido $\rightarrow$ motivação autodeterminada $\rightarrow$ dimensōes da coesão, e 3) Estudar o papel mediador da motivaçâo autodeterminada entre o clima motivacional percebido e a coesão. Participaram no estudo oitocentos e nove jovens jogadores de futebol (798 rapazes e 11 raparigas; $M=11.49$, $D P=1.16)$ que preencheram uma bateria de questionários nos quais se avaliavam as variáveis de interesse no início da temporada. As correlaçôes bivariadas revelam relaçóes positivas entre a percepção de clima de implicação na tarefa e ambas as dimensōes da coesẫo (tarefa e social), enquanto que o clima de implicação no ego apresentou correlaçóes negativas com a coesão de tarefa. Os resultados dos modelos de equaçóes estruturais revelam que o clima-tarefa se associou positivamente com a motivação autodeterminada, enquanto que o clima-ego se relacionou no sentido oposto. A motivaçáo autodeterminada relacionou-se de forma positiva tanto com a coesão de tarefa como com a coesão social. Por último, a motivaçấo autodeterminada actuou como mediador parcial entre o clima de implicaçáo na tarefa e ambas as dimensóes da coesão, e como mediador total entre o clima de implicaçáo no ego e a coesão (tarefa e social). Enfatizam-se as implicações dos comportamentos dos treinadores para a construção da coesão nas equipas e para o desenvolvimento da qualidade da motivação.

Palavras-chave: Adolescentes, Futebol, Clima motivacional, Qualidade da motivação.

\section{Introducción}

Uno de los procesos centrales de la dinámica de los equipos deportivos es la cohesión, entendida como "un proceso dinámico que se refleja en la tendencia de un grupo a no separarse y permanecer unido con la finalidad de conseguir sus obje-

Dirección para correspondencia [Correspodence address]: Isabel Balaguer. Facultad de Psicología. Departamento de Psicología Social. Avda. Blasco Ibáńez, 21. 46010 Valencia (Espańa). E-mail: Isabel.Balaguer@uv.es tivos instrumentales o para la satisfacción de las necesidades afectivas de sus miembros" (Carron, Brawley y Widmeyer, 1998, p. 213).

Contar con grupos cohesionados es altamente valorado en el contexto deportivo por los deportistas y entrenadores, clubes e instituciones deportivas y por todos aquellos interesados en el buen funcionamiento del equipo. Esta valora- 
ción positiva se debe, entre otras razones, a que a lo largo del tiempo la cohesión se ha asociado a importantes beneficios, tanto para el equipo (e.g., mayor rendimiento - Carron, Colman, Wheeler y Stevens, 2002) como para los participantes (e.g., mayor compromiso - Leo, Sánchez-Miguel, SánchezOliva, Amado y García-Calvo, 2011; mayor satisfacción de los miembros - Widmeyer y Williams, 1991). De ahí que uno de los intereses de los investigadores haya sido conocer sus principales características, sus antecedentes y, especialmente, desde un punto de vista aplicado, saber de qué forma el entrenador puede actuar como promotor de la cohesión del equipo.

Carron y sus colegas (Carron, Widmeyer y Brawley, 1985) desarrollaron un modelo de cohesión en el que se propone que el nivel de cohesión de un grupo puede ser evaluado a partir de las percepciones individuales de sus miembros sobre: (a) la atracción personal que sienten hacia el grupo y (b) el grupo como una unidad. Se asume que en esta percepción se incluyen dos facetas, la orientación hacia la tarea del grupo (los objetivos comunes del grupo) y la orientación hacia los aspectos sociales del grupo (desarrollo y mantenimiento de relaciones sociales con el grupo). Así pues, este modelo de cohesión está compuesto por cuatro dimensiones: Integración Grupal hacia la Tarea, Integración Grupal hacia lo Social, Atracción Individual hacia el Grupo en la Tarea, y Atracción Individual hacia el Grupo en lo Social. Basado en esta conceptualización, Carron et al. (1985) desarrollaron el cuestionario de entorno de grupo (GEQ, por sus siglas en inglés) para evaluar las percepciones de cohesión de los deportistas a partir de los 18 años, y con él se ha realizado buena parte de la investigación sobre cohesión en el curso de las últimas décadas en el ámbito del deporte. Recientemente, Eys, Loughead, Bray y Carron (2009a) han elaborado el Cuestionario de Entorno Deportivo para Jóvenes (YSEQ, por sus siglas en inglés) destinado para la población adolescente compuesto por dos dimensiones, la Cohesión de tarea y la Cohesión social; sin tener en cuenta la integración y la atracción del grupo, ya que en sus estudios encontraron que los jóvenes no diferenciaban entre estos dos aspectos (Eys, Loughead, Bray y Carron, 2009a, 2009b).

En la investigación realizada durante las últimas décadas en el contexto deportivo, sobre los antecedentes de la cohesión, se ha subrayado la importancia que tienen los líderes y/o los entrenadores (e.g., Duda y Balaguer, 1999; Carron y Hausenblas, 1998). Duda y Balaguer (1999) desarrollaron un modelo integrado sobre los antecedentes y consecuencias de la conducta del entrenador como líder en el que se sugieren enlaces directos entre el clima motivacional percibido creado por los entrenadores con las respuestas cognitivas, afectivas y conductuales, tanto de los deportistas (constructos personales) como del equipo (constructos grupales). También se defienden relaciones directas entre los constructos personales y grupales.

En este trabajo, se estudian las relaciones del clima moti- vacional percibido creado por el entrenador sobre la cohesión percibida (constructo grupal) y la motivación de los jugadores (constructo personal), sugiriendo que la motivación autodeterminada puede actuar como variable mediadora en esta relación.

El clima motivacional creado por el entrenador, en el marco de la teoría de las metas de logro (AGT, por sus siglas en inglés; Ames, 1992; Dweck, 1986, 1999; Nicholls, 1984, 1989), se define como la atmósfera psicosocial prevalente en un equipo, que se caracteriza por un conjunto de facetas que incluyen tanto la estructura situacional, como los criterios que utilizan los entrenadores en su dinámica con el equipo (Ames, 1992). Para evaluar la percepción que tienen los deportistas sobre el clima creado por el entrenador, Duda y sus colaboradores diseñaron el Cuestionario de Clima Motivacional Percibido en el Deporte-2 (Perceived Motivational Climate in Sport Questionnaire-2; PMCSQ-2; Newton y Duda, 1993; Newton, Duda y Yin, 2000) en el que se distinguen dos dimensiones, clima de implicación en la tarea y clima de implicación en el ego. El clima de implicación en la tarea se caracteriza porque el entrenador enfatiza el aprendizaje cooperativo, valora la mejora y el esfuerzo, y hace ver que cada deportista juega un papel importante en el equipo. Por su parte, en el clima de implicación en el ego el entrenador fomenta la rivalidad entre los miembros del equipo, muestra un reconocimiento desigual hacia los deportistas, y utiliza el castigo como respuesta a los errores. Ambos tipos de climas configuran imágenes distintas sobre el grupo con implicaciones diferentes para la cohesión de sus miembros. En un caso, se fomenta la cooperación entre los miembros del grupo (clima-tarea) mientras que en otro se estimula la rivalidad (clima-ego). En el clima-tarea se favorece la colaboración, se promueve la comunicación interpersonal, la coordinación, así como los sentimientos de similitud como miembros del grupo; sin embargo, en el clima-ego se favorece la desconfianza entre los miembros del grupo, se dificulta la comunicación, la coordinación y la unidad entre ellos. En definitiva, en consonancia con Duda (2001), los componentes estructurales de los climas de implicación en la tarea y de implicación en el ego parecen tener diferentes implicaciones para el desarrollo de la cohesión de equipo.

En estudios anteriores se han analizado las relaciones entre el clima motivacional percibido y la cohesión en el contexto deportivo (e.g., Balaguer, Castillo y Duda, 2003; García-Calvo, Leo, Sánchez, Jiménez y Cervelló, 2008; Heuzé, Sarrazín, Masiero, Raimbault y Thomas, 2006; Eys, Jewitt, Evans, Wolf, Bruner y Loughead, 2013). Balaguer y sus colegas (2003), en su estudio con jóvenes futbolistas españoles varones, utilizando el PMCSQ-2 y el GEQ, encontraron que el clima de implicación en la tarea estaba positivamente relacionado con las cuatro dimensiones de la cohesión en sentido positivo y que el clima de implicación en el ego también 
presentaba las mismas relaciones pero en sentido negativo. García-Calvo et al. (2008), en su investigación con futbolistas varones españoles, utilizando los mismos cuestionarios (PMCSQ-2 y GEQ), encontraron relaciones positivas entre clima-tarea y las cuatro dimensiones de cohesión, mientras que el clima de implicación en el ego estaba negativamente relacionado con las dos dimensiones de cohesión social (atracción e integración) y con una de cohesión de tarea (integración). Por su parte, Heuzé et al. (2006) también analizaron estas relaciones, en dos tiempos distintos de la temporada con los mismos cuestionarios (PMCSQ-2 y GEQ) en la versión francesa, con jugadoras de élite de balonmano y de baloncesto. Los resultados informaron que el clima de implicación en la tarea estaba positivamente relacionado con la cohesión de tarea, mientras que el clima de implicación en el ego presentaba relaciones negativas tanto con la cohesión de tarea como con la cohesión social. Recientemente, en un estudio con jóvenes entre 13 y 17 años que participaban en distintos deportes y actividades, utilizando el Youth Sport Environment Questionnaire (YSEQ; Eys et al., 2009a) y el MCSYS (Smith, Cumming y Smoll, 2008), se encontraron relaciones positivas entre el clima de implicación en la tarea y ambos tipos de cohesión (tarea y social), mientras que el clima de implicación en el ego estaba relacionado negativamente solo con la cohesión de tarea (Eys et al., 2013). En general, estos estudios nos informan que la creación de ambientes de mayor implicación en la tarea favorece la cohesión, mientras que la promoción de climas de implicación en el ego parece dificultarla.

De acuerdo con Heuzé y sus colegas (2006), un paso más en el estudio del clima motivacional como antecedente de la cohesión de equipo podría consistir en explorar los mecanismos que podrían estar actuando en esta relación. Yendo en esa dirección, en este trabajo se estudia la calidad de la motivación como mecanismo mediador entre las dimensiones del clima motivacional percibido creado por el entrenador y la cohesión del equipo. Consideramos que las razones que tienen los jugadores para participar en el deporte están relacionadas con el clima motivacional que crean los entrenadores en sus equipos y que a su vez éstos pueden propiciar una mayor o menor cohesión en el equipo a través de la motivación autodeterminada de sus jugadores.

La teoría de la autodeterminación (SDT por sus siglas en inglés; Deci y Ryan, 1985, 2000) defiende la existencia de un continuo de autodeterminación sobre las razones por las que los deportistas practican deporte, yendo desde los tipos de motivación más autodeterminados (motivación intrínseca) hasta los no autodeterminados (no motivación). Las conductas intrínsecamente motivadas representan el prototipo de las actividades autodeterminadas por uno mismo (dirigidas desde dentro) y son consideradas como las de mayor calidad en la motivación; mientras que las conductas extrínsecamente motivadas vienen determinadas por algo o por alguien (dirigidas desde fuera), y por lo tanto son más bajas en autodeterminación que las motivadas intrínsecamente. Las razones extrínsecas varían en función de su relativa autonomía o autodeterminación, oscilando desde muy baja autodeterminación (regulación externa), pasando por la regulación introyectada y llegando hasta un nivel alto de autodeterminación (regulación identificada e integrada). Finalmente, como ausencia de determinación figura la no motivación (véase Ryan y Deci, 2000) considerada como el tipo de motivación de menor calidad. La SDT defiende que la motivación autodeterminada está asociada a consecuencias cognitivas, emocionales y conductuales positivas (Deci y Ryan, 1985).

Tanto desde la SDT (Deci y Ryan, 1985) como desde la AGT (Ames, 1992; Dweck, 1986, 1999; Nicholls, 1984, 1989) se ha defendido que los factores sociales tienen gran importancia sobre los procesos motivacionales (Balaguer, 2007). Particularmente, desde la AGT se ha considerado que en los contextos sociales en los que se favorece la adquisición de metas tarea (e.g., esfuerzo, maestría) se facilitará la calidad de la motivación (e.g., motivación intrínseca), mientras que en los que se fomentan las metas ego (e.g., ser el mejor) ésta se verá dificultada (e.g. motivación extrínseca). Los resultados de la investigación previa han mostrado apoyo a estas hipótesis encontrándose que el clima de implicación en la tarea estaba asociado positivamente con la motivación intrínseca y negativamente con la no motivación (e.g., Balaguer, Castillo, Duda y García-Merita, 2011; Petherick y Werigard, 2002), mientras que el clima de implicación en el ego estaba relacionado con la motivación extrínseca (e.g., Petherick y Werigard, 2002). En otros trabajos en los que se han estudiado las dos dimensiones del clima motivacional percibido como antecedentes de la motivación autodeterminada se ha visto que el clima de implicación en la tarea está relacionado positivamente con la motivación autodeterminada mientras que el clima de implicación en el ego lo está en sentido negativo (e.g., López-Walle, Balaguer, Castillo y Tristán, 2011; Moreno, Cervelló y González-Cutre, 2010).

Finalmente, la asociación que se propone en este trabajo entre la calidad de la motivación y la cohesión se fundamenta en que las razones que tienen los deportistas para practicar deporte (desde baja hasta alta autodeterminación) pueden favorecer o dificultar la comunicación y coordinación entre los miembros del equipo, lo que a su vez puede promover la cohesión. Por ejemplo, las razones más autodeterminadas (motivación intrínseca, regulación integrada e identificada) conllevan mayor implicación en la tarea, lo que en los deportes de equipo va asociado a una mayor coordinación en el desempeño de la misma, mientras que las menos autodeterminadas (regulación externa e introyectada) y la no motivación reflejan menor interés intrínseco y de menor constancia en la implicación en el quehacer del grupo para la consecución de las metas. En un trabajo previo en el que se ha estudiado la 
relación entre la cohesión (dimensión de Integración Grupal hacia la Tarea) y la motivación autodeterminada, se encontraron relaciones positivas entre estas variables (Blanchard, Amiot, Perreault, Vallerand y Provencher, 2009).

En definitiva, tanto a nivel teórico como empírico la literatura nos informa que la percepción de los climas motivacionales creados por los entrenadores está vinculada con el grado de la calidad de la motivación de los deportistas y que ésta a su vez puede favorecer o dificultar la cohesión del equipo.

Dentro de este marco, en el presente estudio se persiguen tres objetivos: en primer lugar se analizarán, al igual que se ha hecho en estudios anteriores (e.g., Balaguer et al., 2003; García-Calvo et al., 2008; Heuzé et al., 2006: Eys et al., 2013), las relaciones entre las dos dimensiones del clima motivacional percibido creado por el entrenador con las dos dimensiones de la cohesión. Se hipotetiza que las dos dimensiones de la cohesión (tarea y social) estarán relacionadas positivamente con el clima de implicación en la tarea y negativamente con el clima de implicación en el ego. El segundo objetivo consiste en poner a prueba un modelo en el que se hipotetizan las siguientes relaciones: a) la dimensión del clima motivacional de implicación en la tarea estará positivamente relacionada con la motivación autodeterminada; b) la dimensión del clima motivacional de implicación en el ego estará negativamente relacionada con la motivación autodeterminada, y c) la motivación autodeterminada estará positivamente relacionada con las dos dimensiones de la cohesión (tarea y social). En el tercer objetivo se examinará el papel mediador de la motivación autodeterminada entre el clima motivacional percibido creado por el entrenador y las dimensiones de la cohesión.

\section{Método}

\section{Participantes}

Los participantes fueron 809 jóvenes jugadores de fútbol (798 chicos y 11 chicas) con edades comprendidas entre los 9 y los 13 ańos $(M=11.49, D T=1.16)$, pertenecientes a 12 escuelas de fútbol de la Comunidad Valenciana. Los deportista practicaban fútbol una media de 4.46 horas por semana $(D T=1.19)$ y llevaban jugando con su equipo una media de 3.37 temporadas $(D T=2.31)$ en el momento de la recogida de la información.

\section{Instrumentos}

Clima motivacional percibido. Para evaluar las percepciones del clima motivacional creado por el entrenador, los participantes completaron una selección de ítems de la versión española (Balaguer, Mayo, Atienza y Duda, 1997) del Cuestionario de Clima Motivacional Percibido en el Deporte (PMCSQ-2; Newton et al., 2000) adaptados para jóvenes futbolistas. Para el presente estudio se seleccionaron 9 ítems para evaluar el clima de implicación en la tarea (e.g., "Mi entrenador ha valorado a los jugadores que se han esforzado") y 7 ítems para evaluar el clima de implicación en el ego (e.g., "Mi entrenador ha dedicado más atención a los mejores jugadores"). El cuestionario se inicia con la frase: "Durante las últimas 3 ó 4 semanas..." y las respuestas se recogen en una escala tipo Likert de cinco puntos que oscila desde (1) muy en desacuerdo a (5) muy de acuerdo. Este cuestionario ha mostrado una adecuada consistencia interna y validez factorial (Balaguer et al., 1997, Balaguer et al., 2003; Newton et al., 2000).

Regulaciones motivacionales. La motivación fue evaluada con la versión española del Cuestionario de Regulación Conductual en el Deporte (BRSQ-6; Lonsdale, Hodge y Rose, 2008) adaptada para jóvenes futbolistas (Viladrich et al., 2013). En esta escala que consta de 20 ítems, divididos en cinco subescalas, se les solicita a los futbolistas que indiquen en qué medida cada una de las razones expuestas explican por qué juegan al fútbol con su equipo. La escala se inicia con la frase "Juego al fútbol en este equipo...". Ejemplos de ítems para cada una de las subescalas son los siguientes: "porque disfruto" (motivación intrínseca), "porque para mí son importantes los beneficios del fútbol” (regulación identificada), "porque me sentiría culpable si lo dejara" (regulación introyectada), "porque los demás me empujan a hacerlo" (regulación externa), "aunque me pregunto por qué continúo" (no motivación). Las respuestas se recogen en una escala tipo Likert que oscila desde (1) muy en desacuerdo a (5) muy de acuerdo. Investigaciones previas han confirmado la fiabilidad del instrumento (e.g., Lonsdale et al., 2008; Viladrich et al., 2013). Siguiendo el ejemplo de estudios previos (p.e., Balaguer, Castillo y Duda, 2008; Standage, Duda y Ntoumanis, 2003), para operacionalizar la motivación autodeterminada, se utilizó un índice de autodeterminación. Este índice se obtiene calculando el peso que tiene cada tipo de motivación de acuerdo con su posición en el continuo de autodeterminación y sumando el producto. La motivación intrínseca tiene el peso más alto (2), la regulación identificada un peso menor (1), la regulación externa recibe un peso negativo (-1) y la no motivación recibe el peso más negativo $(-2)$. La regulación introyectada representa el punto medio del continuo de la autodeterminación y, por tanto, no se considera en el cálculo del índice de autodeterminación. Valores altos en este índice reflejan una elevada motivación autodeterminada, mientras que valores bajos indican ausencia o baja motivación autodeterminada.

Cohesión de equipo. La percepción que tienen los deportistas de la cohesión se evaluó con la versión castellana del Cuestionario de Entorno Deportivo para Jóvenes (YSEQ; Eys et al., 2009a). Este cuestionario, compuesto por 18 ítems, evalúa la cohesión de tarea (8 ítems; e.g., "Todos hemos esta- 
do igual de comprometidos con los objetivos del equipo") y la cohesión social (8 ítems; e.g., "Algunos de mis mejores amigos están en este equipo”). Los dos ítems restantes se utilizan para identificar la aquiescencia en las respuestas por parte de los participantes pero no se incluyen en el cálculo de las puntuaciones en las dimensiones. Los deportistas responden en una escala tipo Likert que oscila desde (1) muy en desacuerdo a (5) muy de acuerdo. Investigaciones previas han mostrado validez factorial y coeficientes de fiabilidad aceptables (e.g., Eys et al., 2009a; Eys et al., 2013). El YSEQ fue traducido al castellano teniendo en cuenta las guías de la Comisión Internacional de Tests (Hambleton, 2005; véase Duda, 2013).

\section{Procedimiento}

Previo al comienzo de la investigación se obtuvo la aprobación por parte del comité ético de investigación en humanos de la Comisión Ética de la Universitat de València. Se invitó a los líderes de los clubes seleccionados aleatoriamente a participar en el proyecto PAPA (por sus siglas en inglés: Promoting Adolescent Physical Activity, es decir, "Promoviendo la Actividad Física en los Adolescentes”) (para más información véase Duda et al., 2013), así como a los entrenadores, padres y jugadores. Se obtuvo el consentimiento informado de los clubes, de los padres y de los jugadores. Los jugadores cumplimentaron de forma voluntaria y anónima los cuestionarios previamente a una sesión de entrenamiento en sus respectivos clubes con una duración aproximada de 25-45 minutos (dependiendo de la edad y habilidades del jugador). No estuvie- ron presentes los entrenadores ni los directivos de los clubes, y al menos un investigador estuvo presente durante la administración. Para una descripción del protocolo y del procedimiento véase Duda et al. (2013). Los datos fueron recogidos en octubre-diciembre de la temporada 2011/2012.

\section{Resultados}

Los descriptivos y correlaciones de las variables del estudio se presentan en la Tabla 1. Los jugadores informaron percibir un clima de implicación en la tarea alto y un clima de implicación en el ego moderado. Respecto a las regulaciones motivacionales, los jugadores informaron de alto grado de motivación intrínseca y regulación identificada, y de baja regulación introyectada, externa y no motivación. Por último, la cohesión percibida en sus equipos tanto de tarea como social fue alta, siendo más alta la cohesión de tarea.

Las correlaciones bivariadas entre las variables del estudio informaron que el clima-tarea se relacionó positivamente con las razones más autodeterminadas (motivación intrínseca y regulación identificada) y con las dos dimensiones de la cohesión, mientras que la relación fue negativa con las razones controladoras (regulación externa e introyectada) y con la no motivación. Por su parte, el clima-ego se relacionó positivamente con las razones controladoras (regulación externa e introyectada) y con la no motivación y negativamente con las razones más autodeterminadas (motivación intrínseca y regulación identificada) y con la cohesión de tarea.

Tabla 1. Descriptivos y Correlaciones Bivariadas de las Variables del Estudio.

\begin{tabular}{|c|c|c|c|c|c|c|c|c|c|c|c|}
\hline Variables & $M$ & $D T$ & 1 & 2 & 3 & 4 & 5 & 6 & 7 & 8 & 9 \\
\hline 1. Clima Tarea & 4.28 & .58 & - & & & & & & & & \\
\hline 2. Clima Ego & 2.36 & .82 & $-.33^{* *}$ & - & & & & & & & \\
\hline 3. Motivación Intrínseca & 4.61 & .58 & $.43^{* *}$ & $-.17^{* *}$ & - & & & & & & \\
\hline 4. Regulación Identificada & 4.21 & .70 & $.36^{* *}$ & $-.14^{* *}$ & $.51^{* *}$ & - & & & & & \\
\hline 5. Regulación Introyectada & 2.30 & 1.17 & $-.10^{* *}$ & $.29^{* *}$ & $-.08^{*}$ & $.08^{*}$ & - & & & & \\
\hline 6. Regulación Externa & 2.08 & 1.05 & $-.15^{* *}$ & $.29^{* *}$ & $-.20^{* *}$ & .01 & $.60^{* *}$ & - & & & \\
\hline 7. No Motivación & 1.91 & 1.11 & $-.17^{* *}$ & $.25^{* *}$ & $-.25^{* *}$ & $-.09^{*}$ & $.44^{* *}$ & $.61^{* *}$ & - & & \\
\hline 8. Mot Autodeterminada & 8.02 & 3.51 & $.35^{* *}$ & $-.31^{* *}$ & $.64^{* *}$ & $.42^{* *}$ & $-.43^{* *}$ & $-.67^{* *}$ & $-.87^{* *}$ & - & \\
\hline 9. Cohesión Tarea & 4.11 & .71 & $.47^{* *}$ & $-.22^{* *}$ & $.48^{* *}$ & $.42^{* *}$ & -.02 & $-.12^{* *}$ & $-.16^{* *}$ & $.31^{* *}$ & - \\
\hline 10. Cohesión Social & 3.83 & .76 & $.26^{* *}$ & -.05 & $.33^{* *}$ & $.33^{* *}$ & .04 & -.04 & $-.07^{*}$ & $.20^{* *}$ & $.51^{* *}$ \\
\hline
\end{tabular}

Clima motivacional, motivación autodeterminada y cohesión

El modelo de ecuaciones estructurales hipotetizado (véase Figura 1) se analizó utilizando la matriz de correlaciones policóricas como entrada para el análisis de los datos y el mé- todo de Máxima Verosimilitud según está implementado en el programa LISREL 8.80 (Jöreskog y Sörbom, 2006). Se utilizaron los ítems como indicadores de los constructos del modelo. Para la motivación autodeterminada se calcularon cuatro índices que sirvieron como indicadores, utilizando los 
ítems individuales de cada subescala siguiendo la siguiente fórmula: [2 x motivación intrínseca + regulación identificada) - (regulación externa $+2 \mathrm{x}$ no motivación)] (véase Vallerand, 1997). Dicho modelo fue puesto a prueba siguiendo los dos pasos sugeridos por Kline (2005). En el primer paso, se analizó el modelo total de medida donde se estiman los índices de ajuste y los parámetros con todas las variables latentes relacionadas entre sí, lo que resulta equivalente a un modelo factorial confirmatorio, poniendo además a prueba la validez divergente de las variables latentes. El segundo paso consistió en evaluar el modelo de ecuaciones estructurales hipotetizado. Se emplearon diversos índices de ajuste que incluyeron la raíz del promedio del error de aproximación (RMSEA), el índice de ajuste no normativo (NNFI), y el índice de ajuste comparativo (CFI). Valores de RMSEA por debajo de $.08 \mathrm{y}$ de NNFI y CFI superiores a .90 indican un ajuste aceptable de los datos (Hu y Bentler, 1995) sobre todo cuando se analizan modelos complejos con indicadores múltiples (Marsh, Hau y Wen, 2004).

Figura 1. Modelo hipotetizado de relaciones entre las dimensiones del clima motivacional, la motivación autodeterminada y las dimensiones de la cohesión.

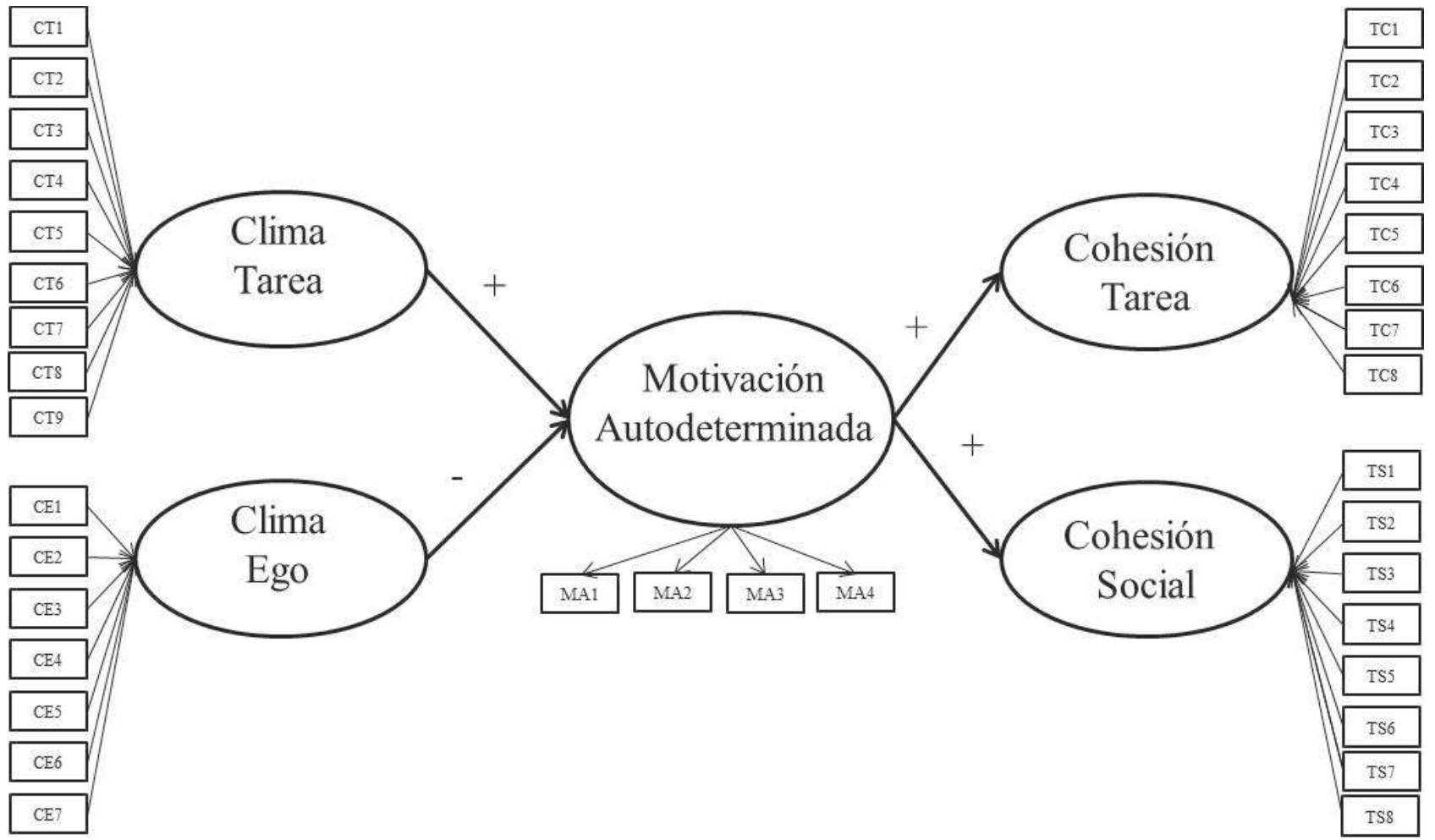

El modelo de medida mostró que cada ítem saturaba adecuadamente en su respectivo constructo latente. Los índices de ajuste fueron adecuados: $c 2(584)=2230.97, p<0.01$; CFI $=0.94 ; \mathrm{NNFI}=0.93 ; \mathrm{RMSEA}=0.069(\mathrm{IC} 90 \%=0.066-$ 0.071).

La fiabilidad de las escalas fue satisfactoria con alfas cuyo rango oscila entre .68 y .83 , a excepción de la escala de regulación identificada cuyo alfa fue de .52 .

El modelo estructural presentó índices de ajuste aceptables: $c 2(588)=2498.30, p<0.01$; CFI $=0.93$; NNFI $=0.93$;
RMSEA $=0.073($ IC 90\% $=0.070-0.075)$. Los coeficientes estandarizados significativos se presentan en la Figura 2. El clima-tarea se asoció positivamente con la motivación autodeterminada, mientras que el clima-ego lo hizo de forma negativa. La motivación autodeterminada se relacionó de forma positiva tanto con la cohesión de tarea como con la cohesión social. Por último, el modelo propuesto explicó el 53\% de la varianza de la cohesión de tarea, el 36\% de la cohesión social y el $20 \%$ de la motivación autodeterminada. 
Figura 2. Solución estandarizada del modelo de relaciones entre las dimensiones del clima motivacional, la motivación autodeterminada y las dimensiones de la cohesión.

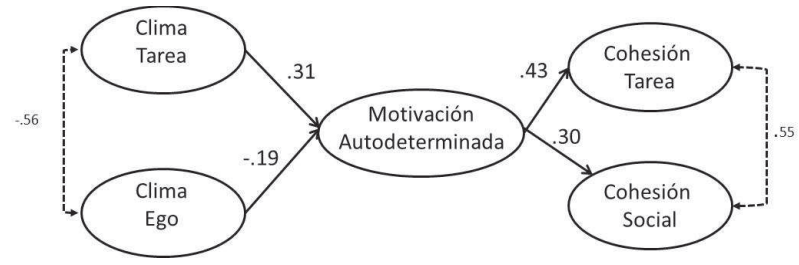

Nota. Todos los coeficientes fueron significativos $p<.01$

\section{Análisis de la mediación de la motivación autodeterminada}

Para analizar la significación de la mediación se calculó el efecto utilizando el método de producto de coeficientes de MacKinnon (2008). El efecto estimado para la motivación autodeterminada entre clima-tarea y cohesión tarea $(\alpha \beta=$ $0.13)$ fue estadísticamente significativo $(P=\mathrm{z} \alpha * \mathrm{z} \beta=27.20, p$ $<0.01)$ al igual que el efecto estimado para la motivación autodeterminada entre clima-tarea y cohesión social $(\alpha \beta=0.09)$ que también fue estadísticamente significativo $\left(P=\mathrm{z} \alpha{ }^{*} \mathrm{z} \beta=\right.$ $18.98, p<0.01)$. El efecto de la motivación autodeterminada entre clima-ego y cohesión de tarea $(\alpha \beta=-0.03)$ fue estadísticamente significativo $(P=\mathrm{z} \alpha * \mathrm{z} \beta=-17.00, p<0.01)$, al igual que el efecto estimado para la motivación autodeterminada entre clima-ego y cohesión social $(\alpha \beta=-0.09)$ que también fue estadísticamente significativo $\left(P=\mathrm{z} \alpha{ }^{*} \mathrm{z} \beta=-47.25, p<\right.$ $0.01)$. Así pues se confirma que la motivación autodeterminada media significativamente el efecto que los climas motivacionales tienen sobre las dimensiones de cohesión.

A continuación se calculó el efecto directo o de no mediación de la motivación autodeterminada entre el clima-tarea y la cohesión de tarea, y entre el clima-tarea y la cohesión social, resultando ambos efectos estadísticamente significativos ( $\tau=.56$ y $\tau=.41, p<.01$ respectivamente), indicando una mediación parcial de la motivación autodeterminada entre el clima-tarea y ambas dimensiones de cohesión. Asimismo, se calculó el efecto directo o de no mediación de la motivación autodeterminada entre el clima-ego y la cohesión de tarea, y entre el clima-ego y la cohesión social, resultando ambos efectos estadísticamente no significativos ( $\tau=-.04$ y $\tau=-.05$, $p>.05$ respectivamente), confirmándose la mediación total de la motivación autodeterminada entre el clima-ego y las dos dimensiones de cohesión.

\section{Discusión}

El primer objetivo del presente trabajo consistió en analizar las relaciones entre el clima motivacional percibido creado por el entrenador y las percepciones de los jóvenes jugadores de fútbol sobre la cohesión del equipo. Los resultados ofrecieron apoyo a la primera hipótesis en la que se defendía que la percepción del clima de implicación en la tarea estaría positivamente relacionada con la cohesión de tarea y con la cohesión social mostrándose en sintonía con los obtenidos por Balaguer et al. (2003), García-Calvo et al. (2008) y Eys et al. (2013) cuyos participantes también fueron jóvenes deportistas, tanto varones futbolistas como en el presente trabajo (Balaguer et al., 2003; García-Calvo et al., 2008), como chicos y chicas participantes en diferentes deportes y actividades (Eys et al., 2013). Sin embargo, cuando la muestra de la investigación es de deportistas de élite, los resultados no coinciden totalmente con los del presente trabajo, ya que en el estudio de Heuzé et al. (2006) con jugadoras de balonmano de élite la percepción del clima de implicación en la tarea se relacionó con la cohesión tarea (Integración Grupal hacia la Tarea) pero no con la cohesión social. Estos resultados parecen indicarnos que en el deporte base cuando el entrenador favorece la cooperación entre los jugadores y una buena atmósfera en la consecución de los objetivos del grupo, eso favorece el deseo de mantener vínculos cercanos entre los otros jugadores, tanto durante la participación deportiva como cuando ésta termina; mientras que en el deporte de élite, donde el interés en el rendimiento es muy importante, este tipo de clima contribuye a que las jugadoras se mantengan unidas en la consecución de sus objetivos en el juego, pero esto no tiene implicaciones en sus relaciones mas allá de la cancha.

La segunda hipótesis de este primer objetivo se cumple parcialmente en nuestro estudio. La percepción del clima de implicación en el ego únicamente se relacionó negativamente con la cohesión de tarea en los jóvenes futbolistas, pero no con la cohesión social. Lo que parece indicarnos que cuando los entrenadores castigan los errores, fomentan la rivalidad entre los miembros del grupo y valoran a unos deportistas más que a otros, producen efectos negativos sobre la coordinación de los jugadores en la ejecución de la tarea, sin embargo, no parece que esto afecte significativamente a las relaciones sociales de los jóvenes futbolistas. En el estudio de Eys et al. (2013) con adolescentes chicos y chicas de diferentes deportes y actividades, también se encontraron las mismas relaciones bivariadas que en el presente estudio. Sin embargo, en los de Balaguer et al. (2003) y García-Calvo et al. (2008) con futbolistas varones adolescentes, y en el de Heuzé et al. (2006) con jugadoras de balonmano de élite, la percepción del clima de implicación en el ego sí que se mostró relacionada negativamente tanto con la cohesión de tarea como con la cohesión social. Estas discrepancias podrían deberse a que en el estudio actual y en el de Eys et al. (2013) se utilizó el cuestionario de cohesión para adolescentes (YSEQ; Eys et al., 2009a), mientras que en los otros mencionados (Balaguer et al., 2003; García-Calvo et al., 2008; Heuzé et al., 2006) la cohesión se evaluó a través del GEQ (Carron et al., 1985). En definitiva, aunque en términos generales se replican las relaciones encontradas en estudios previos entre el clima motiva- 
cional y la cohesión, en estudios futuros seria conveniente utilizar ambos cuestionarios en el mismo estudio para explorar si las diferencias obtenidas entre el clima de implicación en el ego y la cohesión social se deben a la utilización específica de uno u otro instrumento o si hay que atender a otras razones.

En el segundo objetivo los resultados del modelo de ecuaciones estructurales fueron en la dirección esperada, y en ellos se constató que cuando los entrenadores crean climas de implicación en la tarea en los que se fomenta el esfuerzo, la mejora y la cooperación en el aprendizaje, éstos favorecen el desarrollo de la calidad de la motivación en los jugadores. Estos resultados van en la misma línea de trabajos previos en los que se ha visto que los climas de implicación en la tarea están relacionados positivamente con la motivación intrínseca (Balaguer et al., 2011; Petherick y Weigand, 2002) y con formas más autodeterminadas de la motivación (López-Walle et al., 2011; Moreno et al., 2010) y negativamente con la no motivación (Balaguer et al., 2011; Petherick y Weigand, 2002).

Por otra parte, y también en la dirección esperada, la percepción del clima de implicación en el ego fue un predictor negativo de la motivación autodeterminada indicándonos que lo que se fomenta en estos ambientes parece conducir a formas de baja calidad de la motivación ya que las razones por las que los jugadores participan en su deporte son o bien de muy baja autodeterminación (e.g., "porque me siento presionado por los demás para seguir haciéndolo") o de ausencia de autodeterminación (e.g., "lo practico a pesar de que me pregunto para qué sirve”), esto es, razones ajenas a la voluntad de los jugadores. Resultados similares se han encontrado en estudios previos en los que se ha encontrado que los climas de implicación en el ego están positivamente relacionados con tipos característicos de la motivación controlada, como la regulación externa y la regulación introyectada y también con la no motivación (e.g., Petherick y Werigard, 2002).

En definitiva, parece confirmarse que los entrenadores tienen mucho que ver con el tipo de motivación que tienen los jóvenes futbolistas para participar en el fútbol ya que a través de la creación de un clima de implicación en la tarea se favorecen las formas mas adaptativas de la motivación, mientras que mediante un clima de implicación en el ego se fomentan las más desadaptativas (Duda y Balaguer, 2007).

Respecto a las relaciones hipotetizadas entre la motivación autodeterminada y las dimensiones de la cohesión, los resultados van en la dirección esperada ya que la motivación autodeterminada actuó como predictor positivo tanto de la cohesión de tarea como de la cohesión social. Observamos que cuando a los jugadores les gusta jugar al fútbol (motivación intrínseca), esto es, están interesados en el juego por sí mismo, y/o quieren aprender nuevas habilidades (regulación identificada), como por ejemplo mejorar su juego, se facilita la integración en el equipo, tanto en la búsqueda de objetivos comunes como en las relaciones sociales entre los miembros.
La relación entre motivación autodeterminada y cohesión de tarea ya se había encontrado en el estudio de Blanchard y sus colegas (2009) con jóvenes jugadores de baloncesto de ambos sexos. En este estudio, además de aportar evidencia sobre los beneficios de la motivación autodeterminada sobre la cohesión de tarea, también se ha visto que cuando los jóvenes jugadores se mueven por razones autodeterminadas para jugar al fútbol, también favorece la cohesión social, esto es, crea vínculos de unión más allá del campo de fútbol. En definitiva, parece ser que una variable individual como la motivación autodeterminada se relacionan con variables de equipo, esto es, con la cohesión del grupo, lo cual tiene importantes implicaciones para la intervención, ya que nos hace pensar que consiguiendo que se desarrolle la calidad de la motivación de los jugadores podría favorecerse la cohesión del equipo.

Finalmente, en el tercer objetivo, en línea con las sugerencias de Heuzé y sus colaboradores (2006), se hipotetizó que la calidad de la motivación actuaría como variable mediadora entre las dos dimensiones del clima motivacional percibido creado por el entrenador y las dos dimensiones de la cohesión del equipo. Hasta el momento la literatura había explorado las relaciones entre el clima motivacional percibido y las dimensiones de la cohesión, pero no conocíamos la existencia de las variables que podrían estar actuando como mediadoras en esta relación. Los resultados del presente estudio muestran que esta hipótesis se confirma totalmente en el caso del climaego y parcialmente en el clima-tarea.

Conocer que la relación que el clima-ego tiene sobre la cohesión (tarea y social) no es directa sino que depende del grado de la motivación autodeterminada de los jugadores nos muestra un camino a través del cual se establecen los vínculos entre dos variables sobre las que hasta el momento sabíamos que se establecían relaciones negativas. Ahora también conocemos que estas relaciones negativas se deben en parte a que la percepción de este tipo de climas (fomento de la rivalidad entre los jugadores, castigo ante los errores y destacar solo a los mejores) afecta negativamente a la calidad de la motivación de los jugadores (no motivación, regulación externa, regulación introyectada) y que esta falta de interés intrínseco en la ejecución de la tarea dificulta la cohesión del equipo. En estudios anteriores se había ofrecido evidencia de que este tipo de atmósfera social no favorecía una buena coordinación en el juego, en el desempeńo de la tarea del equipo en su conjunto (e.g., "todos hemos estado igual de comprometidos con los objetivos del equipo"), y ahora a esto podemos ańadir que en estos ambientes se fomentan una serie de razones motivacionales para participar en el deporte que dificultan el desarrollo de una buena coordinación y cohesión entre los miembros del grupo, tanto en el campo de futbol como fuera de el.

Por otra parte, conocer que las relaciones positivas del clima de implicación en la tarea sobre la cohesión de tarea y sobre la cohesión social se establecen tanto directamente como a través 
de la motivación autodeterminada aporta apoyo empírico a la teoría de las metas de logro y ofrece guías a seguir en el trabajo con los entrenadores. A través de la creación de este tipo de climas en los que los entrenadores favorecen la comunicación, la cooperación y el esfuerzo hemos visto que se fomenta tanto la cohesión en el juego como después del juego y a su vez se promueven la calidad de la motivación de los jugadores (motivación intrínseca y regulación identificada), lo que contribuye a desarrollar la cohesión en los equipos (tarea y social).

En definitiva, en este estudio podemos concluir que: (1) al igual que en estudios anteriores, hemos confirmado la importancia de la percepción de los climas motivacionales creados por los entrenadores sobre las dimensiones de la cohesión en jóvenes jugadores de fútbol; (2) los climas creados por los entrenadores están relacionados con las razones por las que los jóvenes juegan al fútbol, siendo éstas más autodeterminadas en los climas-tarea y menos autodeterminadas en los climasego; (3) a mayor grado de motivación autodeterminada mayor cohesión en los equipos, tanto de tarea como social; (4) los climas de implicación en la tarea producen tanto directa como indirectamente, a través de la motivación autodeterminada, mayor cohesión, tanto de tarea como social; (5) los climas de implicación en el ego dificultan tanto la cohesión de tarea como la cohesión social a través de la calidad de la motivación de los jugadores.

En el futuro sería conveniente que se realizasen análisis longitudinales con estas variables y a ser posible que se utilizasen técnicas estadísticas multinivel.

\section{Aplicaciones prácticas}

Los resultados del presente estudio tienen importantes implicaciones a nivel aplicado, y animan a la creación de climas de implicación en la tarea y evitar los climas de implicación en el ego por parte de los entrenadores. Cuando los jugadores perciben que los clima motivacionales que crean sus entrenadores tienen alto grado de implicación en la tarea y bajo de implicación en el ego, no solo estarán favoreciendo la calidad de la motivación de sus jugadores sino que además conseguirán desarrollar la cohesión en sus equipos.

En definitiva se podrían diseńar protocolos de intervención para el desarrollo de la cohesión de los equipos a través de la creación de climas motivacionales. Con este tipo de estrategias no se interviene con todo el equipo sino que se trabaja directamente con los entrenadores para que ellos aprendan a crear clima motivacionales que producen beneficios en los jugadores (e.g., motivación autodeterminada) y en el equipo (e.g., cohesión). Actualmente existen ejemplos de intervención en la formación de entrenadores llevados a cabo en diferentes países europeos (véase por ejemplo Duda et al., 2013) que pueden servir de guía en trabajos futuros.

Investigación financiada por la Comisión Europea dentro del Séptimo Programa Marco- Salud-FP/2007-2013 (n²23600) como parte del Proyecto PAPA - www.projectpapa.org

\section{Referencias}

1. Ames, C. (1992). Achievement goals, motivational climate, and motivational processes. En G. C. Roberts (Ed.), Motivation in sport and exercise (pp. 161-176). Champaign, IL: Human Kinetics.

2. Balaguer, I. (2007). Clima motivacional, calidad de la implicación y bienestar psicológico: una propuesta de intervención en equipos deportivos. En A. Blanco y J. Rodríguez Marín (Eds.), Intervención Psicosocial (pp. 135-162). Madrid: Pearson, Prentice Hall.

3. Balaguer, I., Castillo, I. y Duda, J. L. (2003). Interrelaciones entre el clima motivacional y la cohesión en futbolistas cadetes. EduPsykhé, 2, 243-258.

4. Balaguer, I., Castillo, I. y Duda, J. L. (2008). Apoyo a la autonomía, satisfacción de las necesidades, motivación y bienestar en deportistas de competición: Un análisis de la Teoría de la Autodeterminación. Revista de Psicología del Deporte, 17, 123-139.

5. Balaguer, I., Castillo, I., Duda, J. L. y García-Merita, M. (2011). Asociaciones entre la percepción del clima motivacional creado por el entrenador, orientaciones disposicionales de meta, regulaciones motivacionales y vitalidad subjetiva en jóvenes jugadoras de tenis. Revista de Psicología del Deporte, 20, 133-148.

6. Balaguer, I., Mayo, C., Atienza, F. y Duda, J. L. (1997). Factorial validity of the Perceived Motivational Climate in Sport Questionnaire-2 in the case of Spanish elite female handball teams (abstract). Journal of Sport \& Exercise Psychology, 19, 27.

7. Blanchard, C. M., Amiot, C. E., Perreault, S., Vallerand, R. J. y Provencher, P. (2009). Cohesiveness, coach's interpersonal style and psychological needs: Their effects on self-determination and athletes' subjective well-being. Psychology of Sport and Exercise, 10, 545-551. doi:10.1016/j.psychsport.2009.02.005

8. Carron, A. V., Brawley, L. R. y Widmeyer, W. N. (1998). The measurement of cohesiveness in sport groups. En J. L. Duda (Ed.), Advances in sport and exercise psychology measurement (pp. 213-226). Morgantown, WV: Fitness Information Technology.

9. Carron, A. V., Colman, M. M, Wheeler, J. y Stevens, D. (2002). Cohesion and performance in sport: A meta analysis. Journal of Sport \& Exercise Psychology, 24, 168-188. doi:10.1080/026404102317200828

10. Carron, A. V. y Hausenblas, H. A. (1998). Group Dynamics in Sport (2a ed.). Morgantown, WV: Fitness Information Technology.

11. Carron, A. V., Widmeyer, W. N. y Brawley, L. R. (1985). The development of an instrument to measure cohesion in sport teams: The Group Environment Questionnaire. Journal of Sport Psychology, 7, 244-266.

12. Deci, E. L. y Ryan, R. M. (1985). Intrinsic motivation and self-determination in human behavior. New York: Plenum Press.

13. Deci, E. L. y Ryan, R. M. (2000). The "what" and "why" of goal pursuits: Human needs and the self-determination of behavior. Psychological Inquiry, 11, 227-268. doi:10.1207/S15327965PLI1104_01

14. Duda, J. L. (2001). Goals perspectives research in sport: Pushing the boundaries and clarifying some misunderstandings. En G. C. Roberts (Ed.), Advances in motivation in sport and exercise (pp. 129-182). Champaign, IL: Human Kinetics.

15. Duda, J. L. (2013). The conceptual and empirical foundations of Em- 
powering Coaching ${ }^{\mathrm{TM}}$ : Setting the stage for the PAPA project. International Journal of Sport and Exercise Psychology, 11, 311-318. doi:10.1080 /1612197X.2013.839414

16. Duda, J. L. y Balaguer, I. (1999). Toward an integration of models of leadership with a contemporary theory of motivation. En R. Lidor y M. Bar-Eli (Eds.), Sport psychology: Linking theory and practice (pp. 213-230). Morgantown, WV: Fitness Information Technology.

17. Duda, J. L. y Balaguer, I. (2007). The Coach-Created Motivational Climate. En D. Lavalee y S. Jowett (Eds.), Social Psychology of Sport (pp. 117-130). Camping, IL. Human Kinetics.

18. Duda, J. L., Quested, E., Haug, E., Samdal, E., Wold, B., Balaguer, I., Castillo, I., Sarrazin, P., Papaioannou, A., Ronglan, L. T., Hall, H. y Cruz, J. (2013). Promoting Adolescent health through an intervention aimed at improving the quality of their participation in Physical Activity ('PAPA'): Background to the project and main trial protocol. International Journal of Sport and Exercise Psychology, 11, 319-327. doi: 10.1080/1612197X.2013.839413

19. Dweck, C. S. (1986). Motivational processes affecting learning. American Psychologist, 41, 1040-1048. doi:10.1037/0003-066X.41.10.1040

20. Dweck, C. S. (1999). Self-theories: Their role in motivation, personality, and development. Philadelphia: Psychology Press.

21. Eys, M. A., Jewitt, E., Evans, M. B, Wolf, S., Bruner, M. W. y Loughead, T. M. (2013). Coach-initiated motivational climate and cohesion in youth sport. Research Quarterly for Exercise and Sport, 84, 373383. doi:10.1080/02701367.2013.814909

22. Eys, M. A., Loughead, T. M., Bray, S. R. y Carron, A. V. (2009a). Development of a cohesion questionnaire for youth: The Youth Sport Environment Questionnaire. Journal of Sport \& Exercise Psychology, 31, 390-408.

23. Eys, M. A., Loughead, T. M., Bray, S. R. y Carron, A. V. (2009b). Perceptions of cohesion by youth sport participants. The Sport Psychologist, 23, 330-345.

24. García-Calvo, T., Leo, F. M., Sánchez, P. A., Jiménez, R. y Cervelló, E. (2008). Importancia de los aspectos motivacionales sobre el grado de cohesión en equipos de fútbol. Revista Iberoamericana de Psicología del Ejercicio y el Deporte, 3, 61-74.

25. Hambleton, R. K. (2005). Issues, designs and technical guidelines for adapting tests into multiple languages and cultures. En R. K. Hambleton, P. F. Merenda, y C. D. Spielberger (Eds.), Adapting psychological and educational tests for cross-cultural assessment (pp. 3-38). Mahwah, NJ: Lawrence Erlbaum.

26. Heuzé, J. P., Sarrazin, P., Masiero, M., Raimbault, N. y Thomas, J. P. (2006). The relationships of perceived motivational climate to cohesion and collective efficacy in elite female teams. Journal of Applied Sport Psychology, 18(3), 201-218. doi:10.1080/10413200600830273.

27. Hu, L. y Bentler, P. M. (1995). Evaluating model fit. En R. H. Hoyle (Ed.), Structural equation modeling: Concepts, issues, and applications (pp. 76-99). Thousand Oaks, CA: Sage.

28. Jöreskog, K. G. y Sörbom, D. (2006). LISREL 8.80 for Windows [Computer Software]. Lincolnwood, IL: Scientific Software International.

29. Kline, R. B. (2005). Principles and Practice of Structural Equation Modeling (2nd Edition). New York: The Guilford Press.

30. Leo, F. M., Sánchez-Miguel, P. A., Sánchez-Oliva, D., Amado. D. y García-Calvo, T. (2011). Interacción de los niveles de cohesión en el grado de compromiso de jóvenes futbolistas. Movimiento humano, 1 , 13-26.

31. Lonsdale, C., Hodge, K. y Rose, E. A. (2008). The Behavioural Regu- lation in Sport Questionnaire (BRSQ): Instrument development and initial validity evidence. Journal of Sport and Exercise Psychology, 30, 323-355.

32. López-Walle, J., Balaguer, I., Castillo, I. y Tristán, J. (2011). Clima motivacional percibido, motivación autodeterminada y autoestima en jóvenes deportistas mexicanos. Revista de Psicología del Deporte, 20, 149-164.

33. MacKinnon, D. P. (2008). Introduction to statistical mediation analysis. NJ: Mahway.

34. Marsh, H. W., Hau, K. T. y Wen, Z. (2004). In search of golden rules: Comment on hypothesis testing approaches to setting cutoff values for fit indexes and dangers in overgeneralising Hu y Bentler's (1999) findings. Structural Equation Modeling, 11, 320-341. doi:10.1207/ s15328007sem1103_2

35. Moreno, J. A., Cervelló, E. y González-Cutre, D. (2010). The achievement goal and selfdetermination theories as predictors of dispositional flow in young athletes. Anales de Psicología, 26, 390-399.

36. Newton, M. L. y Duda, J. L. (1993). The Perceived Motivational Climate in Sport Questionnaire: Construct and Predictive utility. Journal of Sport \& Exercise Psychology, 15, 5-56.

37. Newton, M. L., Duda, J. L. y Yin, Z. (2000). Examination of the psychometric properties of the Perceived Motivational Climate in Sport Questionnaire-2 in a simple of female athletes. Journal of Sport Science, 18, 275-290. doi:10.1080/026404100365018

38. Nicholls, J. G. (1984). Achievement motivation: Conceptions of ability, subjective experience, task choice, and performance. Psychological Review, 91, 328-346. doi:10.1037/0033-295X.91.3.328

39. Nicholls, J. G. (1989). The competitive ethos and democratic education. Cambridge, MA: Harvard University Press.

40. Petherick, C. M. y Weigand, D. A. (2002). The relationship of dispositional goal orientations and perceived motivational climates on indices of motivation in male and female swimmers. International Journal of Sport Psychology, 33, 218-237.

41. Ryan, R. M. y Deci, E. L. (2000). Self-determination theory and the facilitation of intrinsic motivation, social development, and well-being. American Psychologist, 55, 68-78. doi:10.1037/0003-066X.55.1.68

42. Smith, R. E., Cumming, S. P. y Smoll, F. L. (2008). Development and validation of the Motivational Climate Scale for Youth Sports. Journal of Applied Sport Psychology, 20, 116-136. doi:10.1080/10413200701790558

43. Standage, M., Duda, J. L., y Ntoumanis, N. (2003). A model of contextual motivation in physical education: Using constructs from selfdetermination and achievement goal theories to predict physical activity intentions. Journal of Educational Psychology, 95, 97-110.

44. Vallerand, R. J. (1997). Toward a hierarchical model of intrinsic and extrinsic motivation. En M. Zanna (Ed.), Advances in experimental social psychology, Vol. 29 (pp. 271-360). New York: Academic Press.

45. Viladrich, C., Appleton, P. R., Quested, E., Duda, J. L., Alcaraz, S., Heuzé, J-P., Fabra, P., Samdal, O., Ommundsen, Y., Hill, A. P., Zourbanos, N. y Ntoumanis, N. (2013). Measurement invariance of the Behavioural Regulation in Sport Questionnaire when completed by young athletes across five European countries. International Journal of Sport and Exercise Psychology, 11, 384-394. doi:10.1080/161219 7X.2013.830434

46. Widmeyer, W. N. y Williams, J. M. (1991). Predicting cohesion in a coacting sport. Small Group Research, 22, 548-570. doi:10.1177/1046496491224007 